\title{
Lista de Avaliadores 2021
}

1. Aguinaldo Cesar Fratucci, Universidade Federal Fluminense, RJ

2. Alexandre Augusto Biz, Universidade Federal de Santa Catarina, SC

3. Alice Leoti, Universidade Federal do Pampa, RS

4. Amanda Arrais Mousinho, Universidade de São Paulo, SP

5. Ambrózio Correa de Queiroz Neto, Centro Federal de Educação Tecnológica Celso Suckow da Fonseca, RJ

6. Ana Augusta Ferreira de Freitas, Universidade Estadual do Ceará, CE

7. Ana Cristina Clemente, Universidade de São Paulo, SP

8. Ana Cristina Rempel de Oliveira, Universidade de São Paulo, SP / Universidade Estadual do Oeste do Paraná, PR

9. Ana Paula Sohn, Universidade do Vale do Itajaí, SC

10. Ana Paula Spolon, Universidade Federal Fluminense, RJ

11. Anderson Pereira Portuguez, Universidade Federal de Uberlândia, MG

12. André de Souza Melo, Universidade Federal de Pernambuco, PE

13. Andre Riani Costa Perinotto, Universidade Federal do Delta do Parnaíba, PI

14. Angela Teberga de Paula, Universidade Federal de Tocantins, TO

15. Antônio Carlos Gastaud Maçada, Universidade Federal do Rio Grande do Sul, RS

16. Bruna de Castro Mendes, Instituto Federal de Educação, Ciência e Tecnologia de São Paulo, SP / Universidade Anhembi Morumbi, SP

17. Carlos Alberto Cioce Sampaio, Universidade Regional de Blumenau, SC / Universidade Positivo, PR / Universidade Federal do Paraná, PR

18. Carlos Alberto Freire Medeiros, Universidade Federal do Rio Grande do Norte, RN

19. Carlos Eduardo Silveira, Universidade Federal do Paraná, PR

20. Carolina Todesco, Universidade Federal do Rio Grande do Norte, RN

21. Cassiana Gabrielli, Universidade Federal de São Carlos, SP

22. Claudia Corrêa de Almeida Moraes, Universidade Federal Fluminense, RJ

23. Cláudia Ribeiro de Almeida, Universidade do Algarve, Portugal

24. Clóvis Reis, Universidade Regional de Blumenau, SC

25. Cristina Marques Gomes, Universidade Federal de Santa Maria, RS

26. Cynthia Menezes Mello, Pesquisadora independente, SP

27. Dalila Rosa Hallal, Universidade Federal de Pelotas, RS

28. Daniel Pires Vieira, Universidade de Brasília, DF

29. Daniella Marcondes, Universidade de São Paulo, SP

30. Danielli Cristina Granado, Universidade Estadual Paulista Júlio de Mesquita Filho, SP

31. Diomira Maria Cicci Pinto Faria, Universidade Federal de Minas Gerais, MG 
32. Donária Coelho Duarte, Universidade de Brasilia, DF

33. Dores Cristina Grechi, Universidade Estadual de Mato Grosso do Sul, MS

34. Edar da Silva Añaña, Universidade Federal de Pelotas, RS / Universidade Federal do Rio Grande, RS / Universidade do Vale do Itajaí, SC

35. Edegar Luis Tomazzoni, Universidade de São Paulo, SP

36. Elisangela Aparecida Machado da Silva, Universidade de Brasilia, DF

37. Eloise Silveira Botelho, Universidade Federal do Estado do Rio de Janeiro, RJ

38. Esdras Matheus Matias, Universidade Federal da Paraíba, PB

39. Fabia Trentin, Universidade Federal Fluminense, RJ

40. Fabricio Scarpeta Matheus, University of Northern British Columbia, Canadá

41. Fernando Luiz Araujo Sobrinho, Universidade de Brasilia, DF

42. Francisco Vicente Sales Melo, Universidade Federal de Pernambuco, PE / Faculdade Ateneu, CE / Faculdade Boa Viagem, PE

43. George Bedinelli Rossi, Universidade de São Paulo, SP

44. Glauria Janaina dos Santos, Instituto Federal de Educação, Ciência e Tecnologia da Bahia, BA

45. Guilherme Augusto Pereira Malta, Universidade Federal de Minas Gerais, MG

46. Helena Araujo Costa, Universidade de Brasília, DF

47. Hélio Hintze, Pesquisador independente, SP

48. Heros Augusto Santos Lobo, Universidade Federal de São Carlos, SP

49. Humberto Fois-Braga, Universidade Federal de Juiz de Fora, MG

50. Isabela Barbosa Frederico, Universidade Estadual de Campinas, SP

51. Ivaneli Schreinert Dos Santos, Universidade de São Paulo, SP

52. Izabel Cristina Augusto de Souza Faria, Universidade Federal do Estado do Rio de Janeiro, RJ

53. Izac de Oliveira Belina Bonfim, Universidade Federal do Mato Grosso do Sul, MS

54. Jean Henrique Costa, Universidade Estadual do Rio Grande do Norte, RN

55. Jessica Aline Franco Frazão, Universidade de São Paulo, SP

56. Joao Mendes Rocha, Universidade de Brasília, DF

57. Joice Lavandoski, Universidade Federal do Estado do Rio de Janeiro, RJ

58. Juliana Medaglia, Universidade Federal do Paraná, PR

59. Karina Toledo Solha, Universidade de São Paulo, SP

60. Kerlei Eniele Sonaglio, Universidade Federal do Rio Grande do Norte, RN

61. Kerley Santos Alves, Universidade Federal de Ouro Preto, MG

62. Kettrin Farias Bem Maracajá, Universidade Federal de Campina Grande, PB

63. Laura Castelhano, Pontifícia Universidade Católica de São Paulo, SP

64. Leilianne Michelle Trindade da Silva Barreto, Universidade Federal do Rio Grande do Norte, RN

65. Letícia Bianca Barros de Moraes Lima, Instituto Federal de Educação, Ciência e Tecnologia de Brasilia, DF

66. Lia Sipaúba P. Brusadin, University of Florida, Estados Unidos

67. Liliana Fusco Hemzo, Universidade de São Paulo, SP

68. Linda Maria Rodrigues, Universidade Federal do Maranhão, MA

69. Lucas Goulart, Universidade de São Paulo, SP

70. Luciana Araújo de Holanda, Universidade Federal de Pernambuco, PE 
71. Luciano Torres Tricárico, Universidade do Vale do Itajaí, SC

72. Luiz Carlos da Silva Flores, Universidade do Vale do Itajaí, SC

73. Luiz Eduardo Panisset Travassos, Pontifícia Universidade Católica de Minas Gerais, MG

74. Luiz Gonzaga Godoi Trigo, Universidade de São Paulo, SP

75. Luiz Mendes Filho, Universidade Federal do Rio Grande do Norte, RN

76. Marcelino Castillo Nechar, Universidad Autónoma del Estado de México, México

77. Marcelo Chemin, Universidade Federal do Paraná, PR

78. Marcelo de Oliveira Nunes, Universidade Federal do Rio de Janeiro, RJ

79. Marcelo de Souza Bispo, Universidade Federal da Paraíba, PB

80. Márcia Shizue Massukado-Nakatani, Universidade Federal do Paraná, PR

81. Marcio Luiz MARIETTO, Instituto Politécnico de Leiria, Portugal / Universidade Regional Integrada do Alto Uruguai e das Missões, RS

82. Marconi Costa, Universidade Federal de Pernambuco, PE

83. Marcos Aurelio Tarlombani Silveira, Universidade Federal do Paraná, PR

84. Marcos Eduardo Carvalho Gonçalves Knupp, Universidade Federal de Ouro Preto, MG

85. Maria Angela de Abreu Cabianca, Universidade Anhembi Morumbi, SP

86. Maria da Graça Gomes Ramos, Universidade Federal de Pelotas, RS

87. Maria Henriqueta Sperandio Garcia Gimenes Minasse, Universidade Anhembi Morumbi, SP

88. Mariana Brandâo, Universidade Federal Fluminense, RJ

89. Mariana Bueno de Andrade-Matos, Universidade de São Paulo, SP

90. Mariana Cavalcanti Falcão, Universidade Federal de Pernambuco, PE

91. Mariana Cristina Cunha Souza, Faculdade de Tecnologia de São Paulo, SP

92. Mariana de Freitas Coelho, Universidade Federal de Minas Gerais, MG

93. Mariana Lima, Universidade Federal do Espírito Santo, ES

94. Marina Monteiro da Silva, Universidade de São Paulo, SP

95. Marinês Walkowski, Pesquisadora independente, SC

96. Marklea da Cunha Ferst, Universidade do Estado do Amazonas, AM

97. Marlusa de Sevilha Gosling, Universidade Federal de Minas Gerais, MG

98. Marutschka Martini Moesch, Universidade de Brasília, DF

99. Matheus Belucio, Universidade de Coimbra, Portugal

100. Melise de Lima Pereira, Universidade Federal do Rio Grande, RS

101. Milena Manhães Rodrigues, Universidade de São Paulo, SP

102. Mirian Rejowski, Universidade Anhembi Morumbi, SP

103. Moabe Breno Ferreira Costa, Universidade de São Paulo, SP

104. Moisés Diniz Vassallo, Universidade Federal de Itajubá, MG

105. Natália Tavares Azevedo, Universidade Federal do Paraná, PR

106. Nathália Körössy, Universidade Federal de Pernambuco, PE

107. Pablo Flores Limberger, Universidade do Vale do Itajaí, SC

108. Patricia Cristina Statella Martins, Universidade Estadual de Mato Grosso do Sul, MS

109. Paulo dos Santos Pires, Universidade do Vale do Itajaí, SC

110. Paulo Henrique Assis Feitosa, Universidade de São Paulo, SP 
111. Pedro de Alcântara Bittencourt César, Universidade de Caxias do Sul, RS

112. Priscila Varges da Silva, Universidade Federal do Mato Grosso do Sul, MS

113. Rafaela Almeida Cordeiro, Escola Superior de Propaganda e Marketing, SP

114. Renata Maria Ribeiro, Universidade Estadual Paulista Júlio de Mesquita Filho, SP

115. Ricardo Alexandre Paiva, Universidade Federal do Ceará, CE

116. Ricardo Eustáquio Fonseca Filho, Universidade Federal de Ouro Preto, MG

117. Ricardo Lanzarini, Universidade Federal do Rio Grande do Norte, RN

118. Roberta Abalen Dias, Centro Federal de Educação Tecnológica de Minas Gerais, MG

119. Roberto Meurer, Universidade Federal de Santa Catarina, SC

120. Roberto Pessoa de Queiroz Falcão, Universidade Federal Fluminense, RJ

121. Rodrigo Ladeira, Universidade Federal da Bahia, BA

122. Rodrigo Machado Vilani, Universidade Federal do Estado do Rio de Janeiro, RJ

123. Rodrigo Meira Martoni, Universidade Federal de Ouro Preto, MG

124. Rômulo Duarte, Universidade de São Paulo, SP

125. Rosislene de Fátima Fontana, Universidade Estadual do Oeste do Paraná, PR

126. Sandro Campos Neves, Universidade Federal do Paraná, PR

127. Sarah Minasi, Universidade do Vale do Itajaí, SC

128. Sênia Bastos, Universidade Anhembi Morumbi, SP

129. Sérgio Domingos Oliveira, Universidade Federal Rural do Rio de Janeiro, RJ

130. Sérgio Rodrigues Leal, Universidade Federal de Pernambuco, PE

131. Simone Lira Almeida, Universidade Federal de Pernambuco, PE

132. Stela Cristina Hott Corrêa, Universidade Federal de Minas Gerais, MG

133. Suzana Maria De Conto, Universidade de Caxias do Sul, RS

134. Teresa Cristina Catramby, Universidade Federal Rural do Rio de Janeiro, RJ

135. Thays Cristina Domareski Ruiz, Universidade do Vale do Itajaí, SC

136. Thiago Allis, Universidade de São Paulo, SP

137. Thiago Duarte Pimentel, Universidade Federal de Juiz de Fora, MG

138. Tiago Savi Mondo, Instituto Federal de Educação, Ciência e Tecnologia de Santa Catarina, SC

139. Valeria de Meira Albach, Universidade Estadual de Ponta Grossa, PR

140. Valquíria Padilha, Universidade de São Paulo, SP

141. Vander Valduga, Universidade Federal do Paraná, PR

142. Vânia Lúcia Quadros Nascimento, Universidade Federal do Pará, PA

143. Vera Lucia Bogea Borges, Universidade Federal do Estado do Rio de Janeiro, RJ

144. Verônica Feder Mayer, Universidade Federal Fluminense, RJ

145. Virginia Aparecida Castro, Universidade Federal da Integração Latino-Americana, PR

146. Yolanda Flores e Silva, Universidade do Vale do Itajaí, SC 